\title{
Empatía y tolerancia a la diversidad en un contexto educativo intercultural
}

\section{Empathy and tolerance of diversity in an intercultural educative setting}

Recibido: febrero 28 de 2010 | Revisado: junio 24 de 2011 | Aceptado: diciembre 12 de 2011

\author{
MoIsÈs Esteban-Guitart ** \\ Universidad de Girona, España \\ MARÍA JANE Rivas DAMIÁN *** \\ Universidad Intercultural de Chiapas, México \\ Myriam Rebeca Pérez Daniel ${ }^{* * * * *}$ \\ Universidad Autónoma de Chiapas, México
}

SICI: 1697-9267(201206)11:2<415:ETDCEI>2.0.TX;2-Q

Para citar este artículo. Esteban-Guitart, M., Rivas, M. J. \& Pérez, M. R. (2012). Empatía y tolerancia a la diversidad en un contexto educativo intercultural. Universitas Psychologica, 11(2), 415-426.

* Este trabajo ha sido realizado en el marco de un proyecto de investigación (EDU2009-12875) financiado por el Ministerio de Ciencia e Innovación de España.

** Profesor de tiempo completo del Departamento de Psicología, Facultad de Educación y Psicología, Universidad de Girona, Plaça Sant Domènec, 9, 17006, Girona, España. E-mail: moises.esteban@ udg.edu

**** Secretaría Académica de la Universidad Intercultural de Chiapas, Corral de Piedra, No. 2, Ciudad Universitaria Intercultural, 29299, San Cristóbal de las Casas, Chiapas, México.E-mail: jarima13@ yahoo.com.mx

***** Profesora de tiempo completo adscrita a la Facultad de Ciencias Sociales, de la Universidad Autónoma de Chiapas, San Cristóbal de las Casas, México. E-mail: rebecaperezdaniel@yahoo.com.mx ResearcherID: Pérez, M. F-3573-2012.

\section{RESUMEN}

Las relaciones entre empatía y conducta prosocial han estado ampliamente estudiadas desde hace años. Sin embargo, no existen estudios que utilicen estudiantes indígenas y mestizos de una universidad intercultural. El objetivo principal de la investigación fue analizar la tolerancia a la diversidad en relación a la empatía. La muestra estaba formada por 534 indígenas y mestizos, de edades comprendidas entre los 17 y los 22 años. Los resultados mostraron que los estudiantes con una alta capacidad empática eran también más tolerantes. Las chicas puntuaron significativamente superior en tolerancia y empatía que los chicos. Se encuentran diferencias entre indígenas y mestizos y entre universidad intercultural y universidad pública en relación a áreas específicas de la tolerancia a la diversidad.

Palabras clave autores

Educación intercultural, empatía, tolerancia a la diversidad.

Palabras clave descriptores

Psicología social, pueblos indígenas, investigación cuantitativa.

\section{A B S T R A C T}

The relationship between empathy and prosocial behaviour has been an area of research for many years. Nevertheless, to this day, there is a lack of studies using indigenous and mestizos sample from intercultural university. The main aim of this research was to analyze tolerance of diversity in relation to empathy. The sample consisted of 534 indigenous and mestizos, aged between 17 to 22 years. The results showed that students with high empathic capacity were also more tolerant. Girls scored significantly higher in tolerance and empathy than boys. Differences were found between indigenous and mestizos and between intercultural university and public university regarding tolerance attitudes in specific areas of diversity.

Keywords authors

Intercultural education, empathy, tolerance of diversity.

Keywords plus

Social psychology, indigenous, quantitative research. 


\section{Introducción}

Uno de los rasgos más sobresalientes de las sociedades contemporáneas es la diversidad cultural, étnica, lingüística e identitaria, derivada de los actuales procesos de globalización y migración internacional (Bauman, 1999). En este sentido, la Asociación Americana de Psicología (1993) afirma que "los psicólogos y psicólogas deben reconocer la etnicidad y la cultura como parámetros indispensables para entender los procesos psicológicos" (p. 3). Sin embargo, al igual que pasa con otros constructos sociales, la empatía y la tolerancia a la diversidad son conceptos utilizados a menudo por parte de la opinión pública y distintos organismos políticos, deportivos o religiosos y por ello necesitan ser estudiados sistemáticamente, así como operativizados. En este sentido, el presente estudio pretende contribuir a una mejor comprensión del fenómeno y, a la vez, ser de utilidad en la elaboración de intervenciones educativas interculturales que tienen el propósito de desarrollar actitudes de apertura y respeto hacia la diversidad étnica, lingüística y cultural.

Vázquez (2003) define la tolerancia a la diversidad como el respeto y la consideración hacia las diferencias, como una actitud de aceptación y reconocimiento del pluralismo, incluyendo una dimensión cognitiva o conjunto de creencias preconcebidas respecto a los miembros de otros grupos percibidos como distintos, ya sea por cuestiones de género, etnia, características físicas, lengua, religión. A esta dimensión cognitiva la acompaña una dimensión afectiva vinculada a las valoraciones y reacciones emocionales respecto a los miembros de esos grupos, así como una dimensión conductual vinculada a las acciones en relación con los integrantes de dichos grupos. A pesar de que, según Díaz-Aguado, Segura, Royo y Andrés (1996), la tolerancia es una experiencia psicológica y conductual que se muestra en distintas etapas evolutivas, tiene especial interés en adolescentes y jóvenes ya que en este momento, según distintas investigaciones que los autores revisan, se observa un aumento en la discriminación y el prejuicio hacia otros grupos percibidos como distintos. En lo que hace al contenido de la tolerancia a la diversidad, Lozano y Etxebarria (2007) destacan cuatro dimensiones, a saber: 1) la diversidad nacional, étnica, lingüística o cultural, 2) la diversidad o diferencia física e intelectual, 3) la tolerancia frente a distintas ideologías políticas y, por último, 4) la diferencia relacionada con la clase social.

En relación con la empatía, existen diferentes concepciones de la misma. Davis (1996), tras un riguroso análisis de los orígenes del concepto y sus distintos significados, señala que estas distintas concepciones atañen a una misma realidad ya que se pueden distinguir, al igual que en la tolerancia, distintas dimensiones psicológicas asociadas al fenómeno. En este sentido, el autor propone un modelo multidimensional de la empatía en el que incluye y pone en relación los componentes cognitivo y afectivo de la misma. De manera que hoy en día la mayor parte de los autores coinciden en destacar un componente cognitivo vinculado a la toma de perspectiva (la habilidad para comprender el punto de vista de otra persona), así como un componente afectivo vinculado a la experiencia emocional de sentir lo que el otro siente (Eisenberg, 2000; Hoffman, 2002). En la propuesta original de Davis (1983), el componente cognitivo se traducía en la habilidad de ponerse en el punto de vista o perspectiva de otras personas, así como la tendencia a identificarse con otros personajes de ficción (protagonistas de películas o libros). Mientras que el componente afectivo se operativizaba a través de dos constructos: la preocupación empática y el malestar personal. La preocupación empática alude a un conjunto de sentimientos de preocupación y tristeza ante la necesidad de otras personas; mientras que el malestar personal se refiere a la experiencia de sentimientos de incomodidad y ansiedad cuando uno es testigo de experiencias negativas de otro u otros. En definitiva, según esta línea de investigación, la empatía es una respuesta afectiva y cognitiva de preocupación o compasión ante el sufrimiento ajeno.

En cuanto a la relación entre tolerancia a la diversidad y empatía, un reciente estudio realizado con una muestra de adolescentes concluyó que los participantes que obtenían puntuaciones elevadas en autoestima y en empatía se mostraban más tolerantes. En ese estudio, las chicas obtuvieron 
puntuaciones más altas en empatía y en tolerancia a la diversidad en comparación con los chicos (Lozano \& Etxebarria, 2007). Además del estudio de estos investigadores, otros trabajos muestran que la empatía es un buen predictor de la conducta prosocial traducida en comportamientos tales como compartir, dar apoyo y protección, llevados a cabo voluntariamente para ayudar o beneficiar a otros y otras (Sánchez-Queija, Oliva \& Parra, 2006). De hecho, en las últimas décadas se ha puesto de relieve la importancia de la empatía en la disposición prosocial y el desarrollo moral de las personas, así como su función inhibidora de la agresividad (Eisenberg, 2000; Mestre, Frías \& Samper, 2004; Mestre, Samper \& Frías, 2002). Aspectos que se ponen de manifiesto en aquellas situaciones multiculturales donde es más difícil ponerse en el punto de vista del otro u otra dada la diferencia étnica, lingüística, religiosa, identitaria. En este sentido, no se ha estudiado la empatía, capacidad para comprender al otro y ponerse en su lugar o punto de vista, en contextos de alta diversidad cultural como puede ser una universidad intercultural en la que conviven indígenas y mestizos con distintas lenguas, religiones y procedencias culturales. Lo que nos parece una importante laguna en la investigación clásica alrededor de la empatía y la conducta prosocial.

En este sentido, uno de los ámbitos donde se ha intentado comprender la diversidad cultural con más profundidad es en el terreno educativo, específicamente en el modelo educativo multicultural e intercultural (Banks, 1993; Secretaría de Educación Pública y Coordinación General de Educación Intercultural y Bilingüe [SEP-CGEIB], 2003). Se entiende por educación intercultural el reconocimiento y valoración positiva de la diversidad cultural a través del uso activo de lenguas, saberes y conocimientos de procedencia cultural distinta con el objetivo de contribuir a la cohesión social de un determinado territorio sin excluir la participación de grupos minoritarios (SEP-CGEIB, 2003; Taylor, 2003). Distintos estudios muestran que la participación en un contexto educativo intercultural fomenta la apreciación de la diversidad étnica, lingüística e identitaria, promoviendo conductas prosociales y antirracistas (Banks, 1993). Más concretamente, se ha sugerido que la participación en una universidad intercultural, con un modelo educativo explícito al respecto, combate el racismo y la xenofobia, fomenta la identidad étnica, promoviendo el sentido de comunidad, así como autoconceptos sociales o identidades colectivistas frente a autoconceptos personales o identidades individualistas (Esteban-Guitart, 2011; EstebanGuitart, Rivas \& Pérez, 2011). No obstante, no existen evidencias empíricas del efecto que tiene un modelo educativo superior (universitario) intercultural en medidas asociadas a las actitudes y conductas prosociales como la empatía y la tolerancia a la diversidad, lo que justifica el estudio aquí presentado.

\section{Objetivos e hipótesis de investigación}

El objetivo principal del estudio fue analizar, en una muestra de jóvenes indígenas y mestizos, la relación de la empatía y la tolerancia a la diversidad para poder contrastar cuatro elementos, lo que constituyen los objetivos específicos de la investigación. En primer lugar, y siguiendo estudios previos, la posible relación entre ambos constructos: empatía y tolerancia a la diversidad (Lozano \& Etxebarría, 2007). En segundo lugar, la comparación entre chicos y chicas en relación con las medidas de tolerancia a la diversidad y empatía, dada la relevancia que estudios previos han mostrado en la variable género (Eisenberg, 2000; Garaigordobil $\&$ de Galdeano, 2006; Mestre et al., 2002; Mestre et al., 2004; Pérez-Albéniz, de Paúl, Etxeberría, Montes \& Torres, 2003). En tercer lugar, comparar ambas medidas según la variable condición étnica (indígena frente a mestizo). Aquí no existen estudios previos y sería aventurado plantear alguna hipótesis de investigación. Finalmente, el contraste entre los estudiantes que asisten a una universidad intercultural frente a aquellos que asisten a una universidad no intercultural, con el fin de calibrar el posible impacto que pueda tener dicho modelo educativo sobre la empatía y tolerancia a la diversidad de los estudiantes. En relación con estos cuatro objetivos específicos, planteamos tres hipótesis de investigación, a saber: 
1. Se espera hallar una correlación positiva entre empatía y tolerancia a la diversidad.

2. Se esperan obtener puntuaciones mayores -estadísticamente significativas- en empatía y tolerancia a la diversidad en el grupo de chicas que en el grupo de chicos.

3. Se esperan obtener puntuaciones mayores -estadísticamente significativas- en empatía y tolerancia a la diversidad en el grupo de alumnos de la universidad intercultural en comparación con los alumnos de la universidad no intercultural.

\section{Método}

\section{Participantes}

Participaron en el estudio 534 jóvenes entre los 17 y 22 años de edad ( $M=19.43, D E: 1.964)$, siendo el $51 \%$ chicas (272) frente al restante $49 \%$ chicos (262). Del total, 280 eran mestizos y 254 eran indígenas procedentes de dos universidades públicas de San Cristóbal de las Casas, Chiapas (México), una con un modelo educativo intercultural (un total de 119 mestizos y 131 indígenas) y la otra no (con 161 mestizos y 123 indígenas). Los grupos étnicos fueron identificados mediante una pregunta inicial: "Considero que soy del grupo étnico (marca con una "x")", con dos posibles respuestas: "mestizo/a" e "indígena". El tipo de muestreo fue aleatorio simple ya que se aseguró que cada elemento de la población (estudiantes universitarios de ambas universidades) tuviese la misma probabilidad de ser incluido en la muestra.

\section{Instrumentos}

Los dos instrumentos utilizados fueron: la adaptación española del Interpersonal Reactivity Index de 1983 de Davis (Mestre et al., 2004; Pérez-Albéniz et al., 2003) y el cuestionario de Tolerancia a la Diversidad, realizado por Lozano y Etxebarria (2007).

TABLA 1

Coeficientes de fiabilidad de las subescalas del Índice de Reactividad Interpersonal en distintos estudios

\begin{tabular}{lcl}
\hline & $\begin{array}{c}\text { Coeficiente de } \\
\text { fiabilidad alfa }\end{array}$ & \multicolumn{1}{c}{ Estudio } \\
\hline TP (Toma Perspectiva) & 0.75 & Davis (1983) \\
& 0.75 & Pérez-Albéniz et al. (2003) \\
& 0.56 & Mestre et al. (2004) \\
FS (Fantasía) & 0.62 & Lozano y Etxebarria (2007) \\
& 0.62 & El presente estudio \\
& 0.79 & Davis (1983) \\
PE (Preocupación Empática) & 0.77 & Pérez-Albéniz et al. (2003) \\
& 0.70 & Mestre et al. (2004) \\
& 0.66 & Lozano y Etxebarria (2007) \\
& 0.61 & El presente estudio \\
MP (Malestar Personal) & 0.73 & Davis (1983) \\
& 0.67 & Pérez-Albéniz et al. (2003) \\
& 0.65 & Mestre et al. (2004) \\
& 0.61 & Lozano y Etxebarria (2007) \\
& 0.61 & El presente estudio \\
& 0.75 & Davis (1983) \\
& 0.71 & Pérez-Albéniz et al. (2003) \\
& 0.64 & Mestre et al. (2004) \\
& 0.61 & Lozano y Etxebarria (2007) \\
\hline
\end{tabular}

Fuente: elaboración propia. 
Índice de Reactividad Interpersonal (Mestre et al., 2004)

Para evaluar la empatía se utilizó el Interpersonal Reactivity Index (IRI) de Davis (1983), en su versión y validación española (Mestre et al., 2004; Pérez-Albéniz et al., 2003). Dicho instrumento permite medir las diferencias individuales en las tendencias empáticas, desde un punto de vista multidimensional. Concretamente, el instrumento consta de 28 ítems distribuidos en cuatro subescalas, cada una con siete ítems. Las subescalas corresponden a cuatro constructos que ya hemos presentado en la introducción, pero que a continuación resumimos: Toma de Perspectiva (TP): tendencia o habilidad para adoptar la perspectiva o punto de vista de otras personas; Preocupación Empática (PE): tendencia a experimentar sentimientos de compasión y preocupación hacia otros u otras; Fantasía (FS): tendencia a identificarse con personajes ficticios de libros y películas; y Malestar Personal (MP): experimentar sentimientos de incomodidad y ansiedad cuando uno es testigo de experiencias negativas de otros. La característica más destacada de este instrumento es que permite medir tanto el aspecto cognitivo (TP y FS) como la reacción emocional (PE y MP) del individuo al adoptar una actitud empática. Los participantes tenían que indicar en qué medida cada uno de los ítems les describía adecuadamente en una escala de 5 puntos en la que 1 es nada bien y 5 muy bien. Los coeficientes alfa obtenidos en la muestra fueron de 0.62 en Toma de Perspectiva (TP), 0.61 en Preocupación Empática (PE), 0.61 en Fantasía (FS) y 0.62 en Malestar Personal (MP), obteniendo un coeficiente total de fiabilidad de 0.773 en el total de la muestra estudiada. Se trata de índices bastante modestos, similares a los obtenidos por Mestre et al. (2004) y Lozano y Etxebarria (2007) en muestras españolas, e inferiores que los obtenidos por Pérez-Albéniz et al. (2003), en su adaptación del instrumento al español, y por una de las primeras versiones (1983) de Davis (véase Tabla 1 para una comparativa).
Tolerancia a la Diversidad (Lozano \& Etxebarria, 2007)

Basándose en trabajos anteriores, Lozano y Etxebarria (2007) desarrollan un cuestionario formado por cuatro subescalas, que tiene el propósito de evaluar distintos aspectos vinculados a la tolerancia a la diversidad. Aspectos que ya se han mencionado en la introducción. Estas cuatro subescalas son: 1) Cultura, Etnia, Inmigración (CEI) con 12 ítems (por ejemplo, "me gusta que en mi comunidad residan personas procedentes de muy diferentes lugares -mestizos, indígenas, europeos-"); 2) Características Físicas e Intelectuales (CFI) con 8 ítems (por ejemplo, "tener a compañeros con necesidades educativas especiales en las aulas es positivo para todos y todas"); 3) Ideas Políticas (IP) también con 8 ítems (por ejemplo, "me parece bien que haya diferentes opiniones políticas"); y, por último, 4) Pobreza, Clase Social (PCS), con 6 ítems (por ejemplo, "todas las personas son igualmente válidas: es tan valioso/a el/la que dirige la empresa como el/la que la limpia"). El cuestionario incluye 6 ítems adicionales relacionados con las diferencias de género, la orientación sexual, entre otras. que permiten obtener una escala total de Tolerancia Global a la Diversidad (TGD) formada por 40 ítems (para ver los ítems completos consultar Lozano \& Etxebarria, 2007). En el cuestionario se solicitaba a los participantes que puntuaran su grado de acuerdo o desacuerdo en cada uno de los ítems, siendo el 1 muy en desacuerdo y el 5 muy de acuerdo. En la versión original del instrumento, se obtuvieron los siguientes coeficientes alfa de Cronbach: 0.91 en la TGD, 0.85 en la subescala CEI, 0.70 en la CFI y en la subescala IP y, finalmente, 0.66 en la PCS (Lozano \& Etxebarria, 2007). En muestra del presente estudio se obtuvieron alfas de Cronbach inferiores. A pesar de que la medida total obtiene una puntuación altamente satisfactoria, alfa de 0.866 , las subescalas obtienen puntuaciones de fiabilidad inferiores en comparación con las de la muestra original del cuestionario. En la subescala CEI, un alfa de 0.612; en la subescala CFI, de 0.578; en la IP, de 0.612 y en la PCS, de 0.564. 


\section{Diseño y procedimiento}

Se trata de un estudio descriptivo formado por dos grupos de estudiantes de dos universidades públicas de una misma ciudad. En este sentido, es un estudio descriptivo que sigue el método de encuesta, con un diseño transversal en el que el objetivo es describir una muestra en un momento dado para establecer diferencias entre los distintos grupos que la componen y relaciones entre las variables estudiadas (León \& Montero, 1997). En el presente caso, los grupos de contraste fueron: hombres frente a mujeres, indígenas frente a mestizos y estudiantes de la universidad pública intercultural frente a estudiantes de la universidad pública no intercultural. Las variables analizadas fueron el género: hombre-mujer; la condición etnolingüística: indígena-mestizo; y la participación en un modelo educativo intercultural o no intercultural.

Con relación al procedimiento, dos miembros del equipo de investigación aplicaron los cuestionarios a 20 clases elegidas al azar de alrededor de 30 estudiantes cada una. La administración de los cuestionarios tardó 25 minutos como media. Las instrucciones que se daban eran las siguientes:

A continuación les adjuntamos distintas frases que se refieren a sus pensamientos y sentimientos en una variedad de situaciones, así como una serie de expresiones sobre las que nos gustaría que mostrara su grado de acuerdo o desacuerdo poniendo un número que vaya del 1 (muy en desacuerdo) al 5 (muy de acuerdo). No hay respuestas correctas ni incorrectas, simplemente queremos conocer su opinión en relación con una serie de cuestiones. Rogamos que sean sinceros y sinceras en sus respuestas, así como les garantizamos el anonimato de los cuestionarios poniendo tres letras aleatorias donde debería figurar su nombre. La participación es voluntaria de modo que aquellos o aquellas que no quieran rellenar los cuestionarios no deben hacerlo. Muchas gracias por su colaboración.

Una vez obtenidos los datos empíricos se analizaron mediante el paquete estadístico SPSS versión 15.0. Se realizaron pruebas $t$ para muestras independientes con el objetivo de comparar los resultados obtenidos con otros estudios que han utilizado la misma metodología y las mismas técnicas de análisis de datos, también pruebas $t$ para muestras independientes (Lozano \& Etxebarria, 2007). Se trata de una prueba estadística que, además de que permite comparar datos obtenidos con otros estudios similares, se recomienda su uso cuando se requiere comparar las medias de dos poblaciones independientes (León \& Montero, 1997).

\section{Resultados}

A continuación se agruparon los resultados según los cuatro objetivos específicos de investigación propuestos. En primer lugar, se presentan los resultados en relación con la correlación de la empatía y la tolerancia a la diversidad, así como sus subfactores asociados según los instrumentos administrados. En segundo lugar, analizan las diferencias de género (hombres/mujeres) en los dos cuestionarios. En tercer lugar, se describen los resultados con relación a la comparación empatía y tolerancia a la diversidad según condición étnica (indígenas frente a mestizos). Finalmente, en cuarto lugar, se adjunta la comparación de las medidas empatía y tolerancia a la diversidad según la condición modelo educativo (intercultural frente a no intercultural).

\section{Relación entre empatía y tolerancia a la diversidad}

Con el objetivo de analizar las distintas variables en función de las subescalas de los cuestionarios aplicados se hallaron las correlaciones entre Empatía y sus componentes (Toma de Perspectiva, Fantasía, Preocupación Empática y Malestar Personal) y Tolerancia a la Diversidad y sus componentes o subescalas (Cultura-Etnia-Inmigración, Características Físicas e Intelectuales, Ideas Políticas y Pobreza y Clase Social) (véase Tabla 2).

Como puede observarse en la Tabla 2, se encontraron correlaciones positivas entre todas las variables, siendo todas ellas significativas exceptuando la subescala Fantasía (dentro de la medida de la empatía) con la subescala Pobreza y Clase Social (de la medida de tolerancia a la diversidad), 
TABLA 2

Correlaciones entre las variables Empatía y subcomponentes y Tolerancia a la Diversidad y subcomponentes

\begin{tabular}{lcccccccccc}
\hline & EmpatíaT & TP & FS & PE & MP & DiversidadT & CEI & CFI & IP & PCS \\
\hline EmpatiaT & 1 & & & & & & & & & \\
TP & $0.713^{* * *}$ & 1 & & & & & & & \\
FS & $0.747^{* * *}$ & $0.334^{* *}$ & 1 & & & & & & \\
PE & $0.767^{* *}$ & $0.484^{* *}$ & $0.44^{* * *}$ & 1 & & & & & \\
MP & $0.62^{* *}$ & $0.185^{* *}$ & $0.309^{* *}$ & $0.305^{* *}$ & 1 & & & & \\
DiversidadT & $0.437^{* * *}$ & $0.444^{* *}$ & $0.215^{* * *}$ & $0.418^{* *}$ & $0.163^{* *}$ & 1 & & & \\
CEI & $0.388^{* *}$ & $0.361^{* *}$ & $0.2^{* *}$ & $0.385^{* *}$ & $0.161^{* *}$ & $0.858^{* *}$ & 1 & & \\
CFI & $0.436^{* * *}$ & $0.42^{* *}$ & $0.208^{* *}$ & $0.44^{* *}$ & $0.184^{* *}$ & $0.799^{* * *}$ & $0.571^{* *}$ & 1 & & \\
IP & $0.336^{* *}$ & $0.368^{* *}$ & $0.184^{* * *}$ & $0.279^{* *}$ & $0.11^{* *}$ & $0.781^{* *}$ & $0.569^{* * *}$ & $0.557^{* *}$ & 1 & \\
PCS & $0.272^{* *}$ & $0.323^{* *}$ & 0.086 & $0.285^{* *}$ & 0.082 & $0.785^{* *}$ & $0.606^{* *}$ & $0.577^{* *}$ & $0.487^{* *}$ & 1 \\
\hline
\end{tabular}

${ }^{*} p<0.05 .{ }^{* *} p<0.01$.

EmpatíaT = empatía total; $\mathrm{TP}=$ toma de perspectiva; FS = fantasía; $\mathrm{PE}=$ preocupación empática; $\mathrm{MP}=$ malestar personal; DiversidadT $=$ tolerancia a la diversidad total; CEI = cultura, etnia, inmigración; CFI = características físicas e intelectuales; IP = ideas políticas; PCS = pobreza, clase social.

Fuente: elaboración propia.

TABLA 3

Diferencias de género en el conjunto de variables analizadas

\begin{tabular}{|c|c|c|c|c|c|c|}
\hline & Género & $\mathrm{N}$ & Media & $D E$ & $T$ & $p$ \\
\hline EmpatíaT & $\begin{array}{l}\text { Hombre } \\
\text { Mujer }\end{array}$ & $\begin{array}{l}262 \\
272\end{array}$ & $\begin{array}{l}3.24 \\
3.33\end{array}$ & $\begin{array}{l}0.408 \\
0.407\end{array}$ & -2.29 & $0.022^{*}$ \\
\hline Toma Perspectiva & $\begin{array}{l}\text { Hombre } \\
\text { Mujer }\end{array}$ & $\begin{array}{l}262 \\
272\end{array}$ & $\begin{array}{l}3.5 \\
3.55\end{array}$ & $\begin{array}{l}0.583 \\
0.622\end{array}$ & -0.837 & 0.403 \\
\hline Fantasía & $\begin{array}{l}\text { Hombre } \\
\text { Mujer }\end{array}$ & $\begin{array}{l}262 \\
272\end{array}$ & $\begin{array}{l}3.02 \\
3.08\end{array}$ & $\begin{array}{l}0.584 \\
0.644\end{array}$ & -0.873 & 0.383 \\
\hline Preocupación Empática & $\begin{array}{l}\text { Hombre } \\
\text { Mujer }\end{array}$ & $\begin{array}{l}262 \\
272\end{array}$ & $\begin{array}{l}3.61 \\
3.74\end{array}$ & $\begin{array}{l}4.99 \\
0.532\end{array}$ & -2.54 & $0.01^{* *}$ \\
\hline Malestar Personal & $\begin{array}{l}\text { Hombre } \\
\text { Mujer }\end{array}$ & $\begin{array}{l}262 \\
272\end{array}$ & $\begin{array}{l}2.82 \\
2.96\end{array}$ & $\begin{array}{l}0.557 \\
0.541\end{array}$ & -2.57 & $0.01^{* *}$ \\
\hline Diversidad Total & $\begin{array}{l}\text { Hombre } \\
\text { Mujer }\end{array}$ & $\begin{array}{l}262 \\
272\end{array}$ & $\begin{array}{l}4.06 \\
4.19\end{array}$ & $\begin{array}{l}0.414 \\
0.379\end{array}$ & -3.38 & $0.001^{* * *}$ \\
\hline Cultura, Etnia, Inmigración & $\begin{array}{l}\text { Hombre } \\
\text { Mujer }\end{array}$ & $\begin{array}{l}262 \\
272\end{array}$ & $\begin{array}{l}3.94 \\
4.07\end{array}$ & $\begin{array}{l}0.493 \\
0.446\end{array}$ & -2.88 & $0.004^{* *}$ \\
\hline Características Físicas e Intelectuales & $\begin{array}{l}\text { Hombre } \\
\text { Mujer }\end{array}$ & $\begin{array}{l}262 \\
272\end{array}$ & $\begin{array}{l}4.2 \\
4.34\end{array}$ & $\begin{array}{l}0.476 \\
0.427\end{array}$ & -3.23 & $0.001^{* *}$ \\
\hline Ideas Políticas & $\begin{array}{l}\text { Hombre } \\
\text { Mujer }\end{array}$ & $\begin{array}{l}262 \\
272\end{array}$ & $\begin{array}{l}4.1 \\
4.17\end{array}$ & $\begin{array}{l}0.508 \\
0.462\end{array}$ & -1.53 & 0.127 \\
\hline Pobreza, Clase Social & $\begin{array}{l}\text { Hombre } \\
\text { Mujer }\end{array}$ & $\begin{array}{l}262 \\
272\end{array}$ & $\begin{array}{l}4.27 \\
4.41 \\
\end{array}$ & $\begin{array}{l}0.523 \\
0.501\end{array}$ & -2.81 & $0.005^{* *}$ \\
\hline
\end{tabular}

${ }^{*} p<0.05 .{ }^{* *} p<0.01$.

Fuente: elaboración propia. 
y la correlación Malestar Personal (dentro de la medida de empatía) con Pobreza y Clase Social. Especialmente destacables son las correlaciones obtenidas entre la Empatía Total y sus subescalas (especialmente Preocupación Empática), así como Tolerancia a la Diversidad y sus subescalas (principalmente Cultura, Etnia, Inmigración).

Comparación de las medidas de Empatía y Tolerancia a la Diversidad según la variable género

Para analizar las diferencias de género (mujeres frente a hombres) en las distintas variables consideradas (Empatía y subcomponentes y Tolerancia a la Diversidad y subcomponentes), se aplicaron pruebas $t$ para muestras independientes (véase Tabla 3).

Como se observa en la Tabla 3, todas las puntuaciones media son superiores en el caso de las chicas en comparación con los chicos. Especialmente significativas son las diferencias en las variables
Tolerancia a la Diversidad Total; Cultura, Etnia, Inmigración; Características Físicas e Intelectuales; Pobreza, Clase Social; Malestar Personal; y Problemática Ética. No son significativas las diferencias en las puntuaciones en Toma de Perspectiva, Fantasía e Ideas Políticas.

\section{Comparación de las medidas de Empatía y Tolerancia a la Diversidad según la variable condición étnica (indígena frente a mestizo)}

Para analizar las posibles diferencias entre las variables en función de la condición etnolingüística, indígena frente a mestizo, se realizaron de nuevo pruebas $t$.

Tal y como se muestra en la Tabla 4 solamente se encontraron diferencias estadísticamente significativas en la variable Pobreza, Clase Social (dentro del instrumento que evalúa tolerancia a la diversidad): los indígenas muestran más tolerancia a la pobreza y clase social que los mestizos. Por parte de los in-

TABLA 4

Comparación de las variables analizadas en función de la condición etnolingüística

\begin{tabular}{|c|c|c|c|c|c|c|}
\hline & Género & $\mathrm{N}$ & Media & $D E$ & $T$ & $p$ \\
\hline \multirow[t]{2}{*}{ EmpatíaT } & Mestizo & 280 & 3.26 & 0.422 & -1.09 & 0.273 \\
\hline & Indígena & 254 & 3.31 & 0.4 & & \\
\hline \multirow{2}{*}{ Toma Perspectiva } & Mestizo & 280 & 3.48 & 0.603 & -1.33 & 0.184 \\
\hline & Indígena & 254 & 3.56 & 0.606 & & \\
\hline \multirow[t]{2}{*}{ Fantasía } & Mestizo & 280 & 3.04 & 0.664 & -0.235 & 0.814 \\
\hline & Indígena & 254 & 3.06 & 0.587 & & \\
\hline \multirow[t]{2}{*}{ Preocupación Empática } & Mestizo & 280 & 3.68 & 0.56 & -02.82 & 0.778 \\
\hline & Indígena & 254 & 3.69 & 0.495 & & \\
\hline \multirow[t]{2}{*}{ Malestar Personal } & Mestizo & 280 & 2.85 & 0.522 & 0.832 & 0.406 \\
\hline & Indígena & 254 & 2.93 & 0.569 & & \\
\hline \multirow[t]{2}{*}{ Diversidad Total } & Mestizo & 280 & 4.17 & 0.398 & 1.4 & 0.161 \\
\hline & Indígena & 254 & 4.11 & 0.398 & & \\
\hline \multirow{2}{*}{ Cultura, Etnia, Inmigración } & Mestizo & 280 & 4.04 & 0.459 & 0.832 & 0.406 \\
\hline & Indígena & 254 & 4 & 0.478 & & \\
\hline \multirow[t]{2}{*}{ Características Físicas e Intelectuales } & Mestizo & 280 & 4.27 & 0.484 & -0.415 & 0.679 \\
\hline & Indígena & 254 & 4.29 & 0.432 & & \\
\hline \multirow[t]{2}{*}{ Ideas Políticas } & Mestizo & 280 & 4.19 & 0.487 & 1.55 & 0.121 \\
\hline & Indígena & 254 & 4.11 & 0.478 & & \\
\hline \multirow[t]{2}{*}{ Pobreza, Clase Social } & Mestizo & 280 & 4.31 & 0.495 & 2.16 & $0.031^{*}$ \\
\hline & Indígena & 254 & 4.42 & 0.526 & & \\
\hline
\end{tabular}

${ }^{*} p<0.05$.

Fuente: elaboración propia. 
dígenas, esto se traduce en puntuaciones significativamente más altas en ítems como: "tiendo a tratar de la misma forma al pobre que al rico" ( $M=4.33$; $M=4.05, t=2.579, p<0.01)$. De hecho, en todos los ítems de la subescala Pobreza, Clase Social los indígenas puntúan superior a excepción del ítem invertido 37: "me molesta el tipo de prensa que se aleja de mi ideología política" (indígenas $M=4.51$; mestizos $M=4.58 t=-1.209, p>0.05)$. Es decir, a los mestizos les molesta menos el tipo de prensa que se aleja de su ideología política, en comparación con los indígenas. No obstante, las diferencias no son significativas, siendo casi inexistentes.

\section{Comparación de las medidas de}

Empatía y Tolerancia a la Diversidad según la variable modelo educativo

Finalmente, se comparan las diferencias entre las variables estudiadas en función de la universidad de los y las estudiantes: pública-intercultural frente a pública no intercultural. De nuevo se aplica la prueba $t$ para muestras independientes (véase Tabla 5).

Como se puede observar en la Tabla 5, los estudiantes de la universidad intercultural obtienen medias superiores que los estudiantes de la universidad pública no intercultural en las variables: Empatía Total; Toma de Perspectiva; Fantasía; Diversidad Total; Cultura, Etnia, Inmigración; y Características Físicas e Intelectuales. Sin embargo, las únicas diferencias que son estadísticamente significativas se encuentran en la variable Cultura, Etnia, Inmigración (que forma parte de la escala de Tolerancia a la Diversidad). En cambio los estudiantes de la universidad pública no intercultural (NI) puntúan superior en Problemática Ética; Malestar Personal; Ideas Políticas; y Pobreza, Clase Social. No obstante, las diferencias no son significativas.

\section{TABLA 5}

Comparación de las variables analizadas en función de la universidad

\begin{tabular}{|c|c|c|c|c|c|c|}
\hline & Universidad & $\mathrm{N}$ & Media & $\mathrm{DE}$ & $T$ & $p$ \\
\hline EmpatiaT & $\begin{array}{l}\text { Intercultural } \\
\text { Pública NI }\end{array}$ & $\begin{array}{l}250 \\
284\end{array}$ & $\begin{array}{l}3.29 \\
3.28\end{array}$ & $\begin{array}{l}0.408 \\
0.413\end{array}$ & 0.216 & 0.829 \\
\hline Toma Perspectiva & $\begin{array}{l}\text { Intercultural } \\
\text { Pública NI }\end{array}$ & $\begin{array}{l}250 \\
284\end{array}$ & $\begin{array}{l}3.53 \\
3.51\end{array}$ & $\begin{array}{l}0.604 \\
0.609\end{array}$ & 0.37 & 0.712 \\
\hline Fantasía & $\begin{array}{l}\text { Intercultural } \\
\text { Pública NI }\end{array}$ & $\begin{array}{l}250 \\
284\end{array}$ & $\begin{array}{l}3.09 \\
2.98\end{array}$ & $\begin{array}{l}0.596 \\
0.657\end{array}$ & 1.72 & 0.084 \\
\hline Preocupación Empática & $\begin{array}{l}\text { Intercultural } \\
\text { Pública NI }\end{array}$ & $\begin{array}{l}250 \\
284\end{array}$ & $\begin{array}{c}3.68 \\
3.7\end{array}$ & $\begin{array}{c}0.518 \\
0.53\end{array}$ & -0.331 & 0.74 \\
\hline Malestar Personal & $\begin{array}{l}\text { Intercultural } \\
\text { Pública NI }\end{array}$ & $\begin{array}{l}250 \\
284\end{array}$ & $\begin{array}{l}2.87 \\
2.95\end{array}$ & $\begin{array}{l}0.555 \\
0.544\end{array}$ & -1.32 & 0.188 \\
\hline Diversidad Total & $\begin{array}{l}\text { Intercultural } \\
\text { Pública NI }\end{array}$ & $\begin{array}{l}250 \\
284\end{array}$ & $\begin{array}{l}4.15 \\
4.09\end{array}$ & $\begin{array}{c}0.39 \\
0.412\end{array}$ & 1.59 & 0.112 \\
\hline Cultura, Etnia, Inmigración & $\begin{array}{l}\text { Intercultural } \\
\text { Pública NI }\end{array}$ & $\begin{array}{l}250 \\
284\end{array}$ & $\begin{array}{l}4.32 \\
4.21\end{array}$ & $\begin{array}{l}0.421 \\
0.504\end{array}$ & 2.47 & $0.014^{*}$ \\
\hline Características Físicas e Intelectuales & $\begin{array}{l}\text { Intercultural } \\
\text { Pública NI }\end{array}$ & $\begin{array}{l}250 \\
284\end{array}$ & $\begin{array}{l}4.05 \\
3.96\end{array}$ & $\begin{array}{c}0.464 \\
0.48\end{array}$ & 1.78 & 0.075 \\
\hline Ideas Políticas & $\begin{array}{l}\text { Intercultural } \\
\text { Pública NI }\end{array}$ & $\begin{array}{l}250 \\
284\end{array}$ & $\begin{array}{l}4.14 \\
4.15\end{array}$ & $\begin{array}{l}0.462 \\
0.522\end{array}$ & -0.075 & 0.94 \\
\hline Pobreza, Clase Social & $\begin{array}{l}\text { Intercultural } \\
\text { Pública NI }\end{array}$ & $\begin{array}{l}250 \\
284 \\
\end{array}$ & $\begin{array}{l}4.35 \\
4.36 \\
\end{array}$ & $\begin{array}{l}0.51 \\
0.529 \\
\end{array}$ & -0.184 & 0.854 \\
\hline
\end{tabular}

${ }^{*} p<0.05$.

Fuente: elaboración propia. 


\section{Discusión}

El objetivo general de este estudio era analizar la empatía y la tolerancia a la diversidad en una muestra de indígenas y mestizos de Chiapas no estudiada hasta el momento. Los resultados obtenidos apoyan una asociación significativa de las variables analizadas en este estudio. Solamente la subescala Pobreza, Clase Social (dentro de la medida de tolerancia a la diversidad) no correlaciona, de un modo estadísticamente significativo, con las subescalas Fantasía y Malestar Personal (dentro de la medida de empatía). En sintonía con estudios previos (Lozano \& Etxebarria, 2007), estos resultados ponen de manifiesto que los individuos capaces de ponerse en el punto de vista de otros $\mathrm{u}$ otras (obtienen puntuaciones positivas en empatía), tienden a mostrar actitudes de mayor tolerancia a la diversidad, tanto en general como respecto a las personas de otras culturas, etnias, ideas políticas y características físicas o psíquicas. Especialmente en la presente muestra, aquellos y aquellas que obtienen puntuaciones positivas en tolerancia a la diversidad, obtienen puntuaciones positivas en tolerancia a la diversidad cultural, étnica, inmigración. De la misma manera, aquellas personas que puntúan positivamente en empatía lo hacen en toma de perspectiva, fantasía, malestar personal y, especialmente, preocupación empática -dimensión afectiva de la empatía- (Mestre et al., 2004). De modo que estos resultados son consistentes con la investigación que apoya la asociación entre la empatía y la tolerancia a la diversidad (Lozano \& Etxebarria, 2007). Ello es fácilmente explicable con base en la documentación empírica que apoya una correlación entre la empatía y la conducta prosocial (Eisenberg, 2000; Mestre et al., 2002; Sánchez-Queija et al., 2006), de modo que podríamos considerar la tolerancia a la diversidad como factor vinculado con la empatía y la conducta prosocial. Ser respetuoso con el otro u otra, más allá de su diferencia étnica, de género, física, intelectual podría requerir habilidades también implicadas en la empatía y en la conducta prosocial, aunque la relación entre tolerancia a la diversidad y conducta prosocial aún está por determinar. En este sentido, futuras investigaciones podrían incluir medidas de conducta prosocial para calibrar la relación con la tolerancia a la diversidad. En definitiva, el presente estudio permite apoyar empíricamente la primera hipótesis inicial según la cual se esperaban correlaciones positivas y significativas entre las variables analizadas en el estudio.

En relación con la segunda hipótesis, la mayor puntuación en chicas que en chicos en las medidas de empatía y tolerancia a la diversidad, los resultados obtenidos no permiten rechazarla. Es decir, se muestran consistentes con la literatura previa que muestra una mayor puntuación en empatía por parte de las mujeres, en comparación con los hombres (Davis, 1983; Eisenberg, 2000; Garaigordobil \& de Galdeano, 2006; Mestre et al., 2002; Mestre et al., 2004; Pérez-Albéniz et al., 2003; Sánchez-Queija et al., 2006), y una mayor puntuación también por parte de las mujeres en comparación con los hombres, en tolerancia a la diversidad (Lozano \& Etxebarría, 2007).

Más inesperados son los resultados obtenidos en relación con la tercera hipótesis, aunque en este caso no es de extrañar dado que no existe literatura previa al respecto. En lo que hace a la comparación entre indígenas frente a mestizos, uno de nuestros objetivos específicos -pero sin ninguna hipótesis previa de antemano-, solamente se encuentran diferencias estadísticamente significativas en la subescala de tolerancia a la diversidad: Pobreza, Clase Social. Este dato podría explicarse por razones socioeconómicas y estructurales. En la sociedad chiapaneca, el indígena ocupa lugares destacados en pobreza, en comparación con el mestizo, y clase social minoritaria de modo que podrían ser más sensibles a dichos aspectos, mostrando más tolerancia al respecto. Sin embargo, las diferencias en empatía y sus subfactores y tolerancia a la diversidad y sus subescalas no son concluyentes ni significativas, por lo que deben ser analizadas en futuras investigaciones con el objetivo de descartar, o no, posibles diferencias basadas en la condición etnolingüística. La imagen que se desprende de los resultados es cierta homogeneización en las respuestas, más allá de la condición etnolingüística de los estudiantes. Ello parece no estar en la línea de otras investigaciones que muestran claras diferencias entre indígenas 
y mestizos en otras variables como la identidad étnica, el autoconcepto o la autoestima (EstebanGuitart, 2010; Esteban-Guitart et al., 2011).

Finalmente, el análisis de las diferencias entre los estudiantes de la universidad pública intercultural y de la universidad pública no intercultural en el conjunto de variables consideradas, proporciona resultados interesantes aunque parcialmente inesperados en relación a nuestra hipótesis de partida (la tercera hipótesis), una mayor puntuación en empatía y tolerancia a la diversidad por parte de los estudiantes de la universidad intercultural. A pesar de que en líneas generales los estudiantes de la universidad intercultural puntúan superior en empatía y tolerancia a la diversidad y sus subescalas respectivas, las diferencias solamente son significativas en el factor Cultura, Etnia, Inmigración. Ello indica que los estudiantes de la universidad intercultural son más tolerantes y sensibles a la diversidad étnica y cultural. Estos resultados pueden tener una doble lectura. Por una parte, podríamos suponer que un modelo educativo intercultural fomenta el reconocimiento de la diversidad étnica y cultural, facilitando conductas prosociales y contrarias a la xenofobia y al racismo. Un currículo multicultural o intercultural tiene el objetivo, precisamente, de fomentar el aprecio, la tolerancia, el reconocimiento y la estima de la diversidad cultural, ya sea lingüística, étnica, identitaria, religiosa (Banks, 1993; SEPCGEIB, 2003). Se necesitan futuras investigaciones de naturaleza longitudinal para aclarar el impacto que pueda tener un modelo educativo intercultural en la empatía y la tolerancia a la diversidad.

En definitiva, el presente estudio arroja nuevos datos alrededor de dos constructos vinculados al desarrollo moral y prosocial, la empatía y la tolerancia a la diversidad, con una muestra hasta el momento no estudiada. La fiabilidad de los instrumentos parece ser aceptable con un alfa de Cronbach de 0.773, para la escala de empatía y un 0.866 para la escala de tolerancia a la diversidad. A pesar de que, en líneas generales, las diferencias no son significativas entre indígenas y mestizos y estudiantes de la universidad intercultural en comparación con los estudiantes de la universidad no intercultural, si que merece destacar las diferencias entre chicas y chicos, a favor de las primeras, y las correlaciones positivas y significativas entre las subescalas. Somos conscientes de las limitaciones del estudio, no solo por su carácter correlacional y por los posibles sesgos de deseabilidad social de las medidas de autoinforme utilizadas, sino también porque los datos del mismo provienen de un contexto determinado -estudiantes universitarios- por lo que cabría (sería una posibilidad) encontrar diferencias entre indígenas y mestizos no universitarios, lo que parece desprenderse en relación con la construcción de la identidad de otros estudios (Esteban-Guitart, 2011). Ello obliga a ser muy cautos en la generalización de los resultados, que ni siquiera se pensó en plantear. No obstante, se puede sugerir la conveniencia de prestar atención al desarrollo de la sensibilidad empática y la tolerancia a la diversidad en un contexto internacional marcado por la globalización y la heterogeneidad de las formas de vida (religión, lengua, nacionalidad, etc.), hoy puestas en reunión en un mismo territorio.

\section{Referencias}

Asociación Americana de Psicología. (1993). Guidelines for providers of psychological services to ethnic, linguistic, and culturally diverse populations. American Psychologist, 48(1), 43-45.

Banks, J. A. (1993). Multicultural education for young children: Racial and ethnic attitudes and their modification. En B. Spodek (Ed.), Handbook of research on the education of young children (pp. 236250). New York: Macmillan.

Bauman, Z. (1999). La globalización: consecuencias humanas. México: Fondo de Cultura Económica.

Davis, M. H. (1983). Measuring individual differences in empathy: Evidence for a multidimensional approach. Journal of Personality and Social Psychology, 44(1), 113-126.

Davis, M. H. (1996). Empathy: A social psychological approach. Oxford: Westview Press.

Díaz-Aguado, M. J., Segura, M. P., Royo, P. \& Andrés, M. (1996). Programas de educación para la tolerancia y prevención de la violencia en los jóvenes. Madrid: Instituto de la Juventud, Ministerio de Trabajo y Asuntos Sociales. 
Eisenberg, N. (2000). Emotion, regulation, and moral development. Annual Review of Psychology, 51(1), 665-697.

Esteban-Guitart, M. (2010). Propiedades psicométricas de la Escala de Identidad Étnica Multigrupo en español. Revista Latinoamericana de Psicología, 42(3), 405-412.

Esteban-Guitart, M. (2011). Ethnic identity in an intercultural geography: An empirical study. Canadian Social Science, 7(1), 64-71.

Esteban-Guitart, M., Rivas, M. J. \& Pérez, M. R. (2011). Identidad étnica y autoestima en jóvenes indígenas y mestizos de San Cristóbal de las Casas (Chiapas, México). Acta Colombiana de Psicología, 14(1), 99-108.

Garaigordobil, M. \& de Galdeano, P. G. (2006). Empatía en niños de 10 a 12 años. Psicothema, 18(2), 180-186.

Hoffman, M. L. (2002). Desarrollo moral y empatía. Barcelona: Idea Books S. A.

León, O. G. \& Montero, I. (1997). Diseño de investigaciones. Madrid: McGraw-Hill.

Lozano, A. M. \& Etxebarria, I. (2007). La tolerancia a la diversidad en los adolescentes y su relación con la autoestima, la empatía y el concepto del ser humano. Infancia y Aprendizaje, 30(1), 109-129.
Mestre, M. V., Frías, M. D. \& Samper, P. (2004). La medida de la empatía: análisis del Interpersonal Reactivity Index. Psicothema, 16(2), 255-260.

Mestre, M. V., Samper, P. \& Frías, M. D. (2002). Procesos cognitivos y emocionales predictores de la conducta prosocial y agresiva: la empatía como factor modular. Psicothema, 14(2), 227-232.

Pérez-Albéniz, A., de Paúl, J., Etxeberría, J., Montes, M. P. \& Torres, E. (2003). Adaptación del Interpersonal Reactivity Index (IRI) al español. Psicothema, 15(2), 267-272.

Sánchez-Queija, I., Oliva, A. \& Parra, A. (2006). Empatía y conducta prosocial durante la adolescencia. Revista de Psicología Social, 21(3), 259-271.

Secretaría de Educación Pública \& Coordinación General de Educación Intercultural y Bilingüe. (2003). Universidad Intercultural. Modelo educativo. México: Coordinación General de Educación Intercultural y Bilingüe.

Taylor, C. (2003). El multiculturalismo y la "política del reconocimiento". Madrid: Fondo de Cultura Económica.

Vázquez, A. (2003). Tolerancia: idebilidad o fortaleza? Madrid: Témpora. 